\title{
Supporting information: Using strand displacing polymerase to program chemical reaction
}

networks

Shalin Shah, ${ }^{\dagger+\ldots}$ Jasmine Wee, ${ }^{\S}$ Tianqi Song,,+ Luis Ceze, ${ }^{\S}$ Karin Strauss,, Yuan-Jyue Chen, ${ }^{*}, \mathbb{\pi}$ and John Reif*,+,

$\dagger$ Department of Electrical $\& 3$ Computer Engineering, Duke University, Durham, NC - 27701 $\ddagger$ Department of Computer Science, Duke University, Durham, NC - 27701

\Microsoft Research, Redmond, WA - 98052

$\S$ Paul G. Allen School of Computer Science and Engineering, University of Washington, Seattle, Washington, 98195

E-mail: yuanjc@microsoft.com; reif@cs.duke.edu

\section{Supplementary Methods}

\section{$1.1 \quad$ Materials}

All the DNA strands used in this work were ordered from Integrated DNA Technologies (Coralville, IA). The unmodified strands used in this work were ordered using standard desalting purification. The modified DNA strands with either a fluorophore (Fluorescien; 56FAM) or a quencher (Black hole quencher; 3BHQ_1 and 3BHQ_2) were ordered using HPLC purification. The strand displacing polymerase enzyme Bst 3.0 (Catalog\# M0374S) was ordered from New England Biolabs (Ipswich, MA). Isothermal amplification buffer (Catalog\# 
B0374S), magnesium sulfate (Catalog\# B1003S) and deoxynucleoside triphosphate (dNTP; Catalog\# N0447S) were also ordered from New England Biolabs. Note that dNTP master mix contained high concentration $(100 \mathrm{mM})$ of each base. All the DNA strands were ordered in lyophilized form and were resuspended at $100 \mu \mathrm{M}$ in Tris-EDTA buffer (1x TE) and stored at $-20^{\circ} \mathrm{C}$.

\subsection{DNA sequence design and purification}

The design for DNA sequences followed PCR primer design rules. Each domain was kept $25 \mathrm{nt}$ long, along with $40-60 \% \mathrm{GC}$ content and melting temperature above $60{ }^{\circ} \mathrm{C}$. The $5^{\prime}$ clamps were kept between 5 - $10 \mathrm{nt}$ long and overhang sequences were poly- T tails.

All the unmodified synthetic DNA used in this work was gel purified using 10\% denaturing gel upon arrival at $200 \mathrm{~V}$ for 15 - 30 minutes to remove the truncation errors. The gel

bands were cut carefully and eluted in $1 \mathrm{x}$ TE (or 1x TAE) overnight to recovered most of the DNA. For annealing double-stranded DNA gates, we heat up strands to $95{ }^{\circ} \mathrm{C}$ for 5 minutes followed by slow cooling rate of $1{ }^{\circ} \mathrm{C}$ per minute until $25{ }^{\circ} \mathrm{C}$ is reached; the overall process approximately takes an hour. The annealed gates were purified again using $12 \%$ non-denaturing gel at $150 \mathrm{~V}$ for 5 hours using $1 \mathrm{x} \mathrm{TE} \mathrm{Mg}^{+2}$ as the loading buffer. Similar to denaturing gel, the bands were carefully excised and eluted in $1 \mathrm{x} \mathrm{TE} \mathrm{Mg}^{+2}$ overnight to recover DNA from gel.

\subsection{Master mix for polymerase reactions}

Strand displacing activity of polymerase enzymes such as Bst 3.0 is highly dependent on the environmental conditions such as temperature. To perform fluorescence experiments, we prepared a master mix which contains all the required chemicals and incubated it for 20 minutes at $50^{\circ} \mathrm{C}$ in the plate reader. A typical master mix consists of dsDNA gates, $\mathrm{Mg}^{+2}$ (8 nM), isothermal amplification buffer (1.0x), dNTPs (1 mM), reporter complex, DI water and Bst 3.0 polymerase $(20 \mathrm{U} / \mathrm{ml})$. The total volume was always kept constant at $100 \mu \mathrm{l}$ and 
input strands were added post-incubation of 20 minutes at room temperature. However, the amount of input was always less than $3 \mu \mathrm{l}$ and it was added using a multi-channel pipette to keep the overall process under 30 seconds. This ensured minimal temperature fluctuations upon input addition.

\subsection{Fluorescence data normalization}

All the fluorescence data in this work was collected using Synergy HTX multi-mode plate reader (BioTek Instruments Inc, Vermont). The collected data was exported as a csv file and imported into a jupyter notebook where it was normalized using python scripts. First, the numerical zero for time-axis was set to when the input was added. Second, the y-axis for all the fluorescence curves were offset by some value so the minimum value is subtracted from them to bring the minimum to numerical zero. Finally, depending on reporter concentration the curves are normalized between 0 and 1, and then scaled back up to the maximum value of the reporter concentration.

\section{Strand displacement mechanism}

The strand displacement method using polymerase enzyme is slightly different than toehold mediated strand displacement (TMSD). In TMSD, a short exposed region, called the toehold, makes the strand displacement process slightly energetically favorable. Therefore, the incumbent strand leads to branch migration upon a tug-of-war. Polymerase-based strand displacement (PSD) uses DNA hybridization between primer and template region (or toehold region) for polymerase activation. This step is reversible process, however after polymerase starts priming the process is irreversible and invariably leads to strand displacement of incumbent strand, as shown in Fig. S1b. The red blob in the figure indicates polymerase

enzyme. Finally, there are several ways to represent DNA however, in this work, since we operate at the domain level, we use the third representation in Fig. S1a. 
a

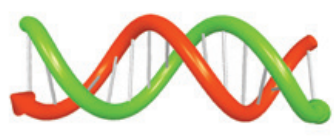

b
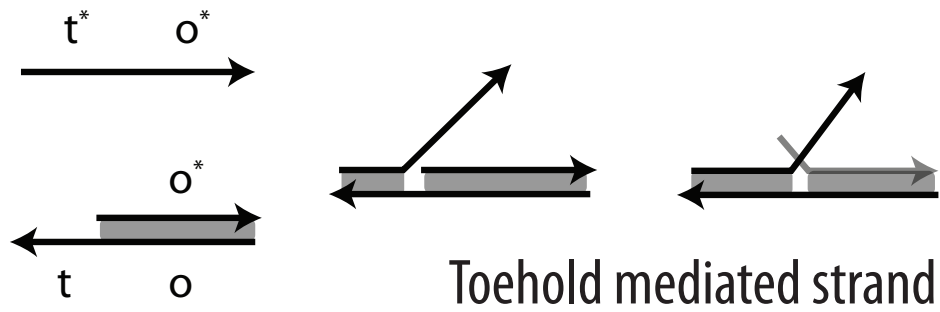

Toehold mediated strand displacement
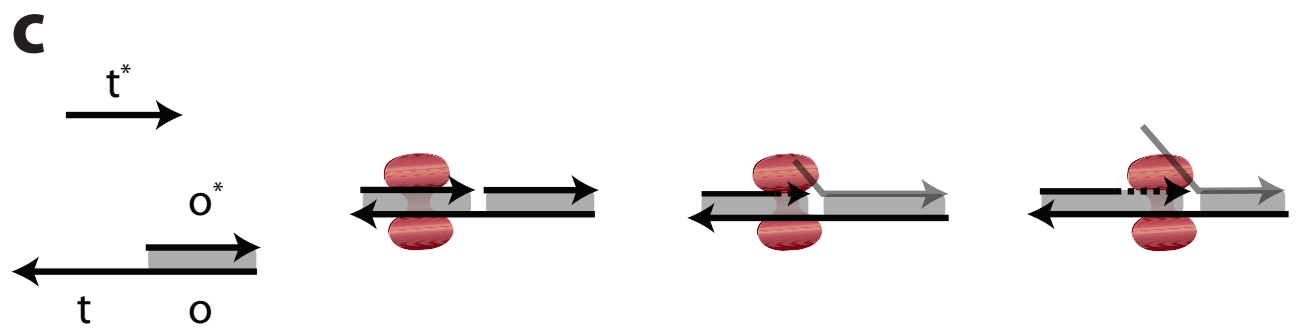

Polymerase-based strand displacement

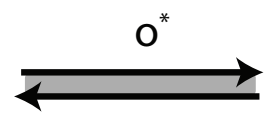

o
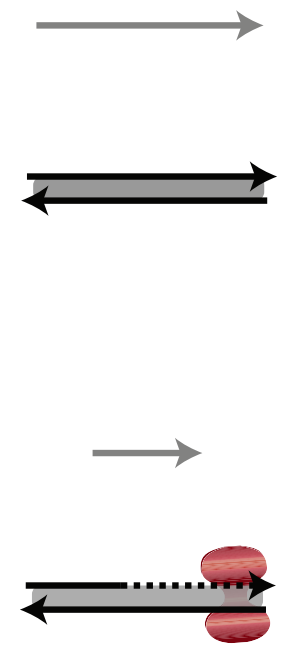

Figure S1: a Abstract representation of DNA. b Toehold-mediated strand displacement. c Polymerase-based strand displacement process.

\section{DNA design for CRN implementation}

It is possible to reduce complex CRNs to a simplified unimolecular and bimolecular form ${ }^{1}$ therefore, we design DNA implementations of these fundamental CRNs. In order to implement a bimolecular reaction $\mathrm{A}+\mathrm{B} \stackrel{\mathrm{k}}{\longrightarrow} \mathrm{C}$, we perform a two-step ${ }^{2}$ process as shown in supplementary Fig. S2b. The input strand A combines with input B (gated form) to produce intermediate strand BI. This intermediate strand can combine with an auxiliary gate IC to produce output $\mathrm{C}$. If unimolecular $\mathrm{CRN}$ is to be implemented such as $\mathrm{A} \stackrel{\mathrm{k}}{\longrightarrow} \mathrm{C}$ The highlighted gate B in supplementary Fig. S2b can become simply an auxiliary gate. Such two-step process is required for a generalized CRN framework since a one-step CRN design, for example, cannot implement reactions such as $\mathrm{A} \stackrel{\mathrm{k}}{\longrightarrow} \mathrm{A}+\mathrm{A}$. This is because dsDNA 

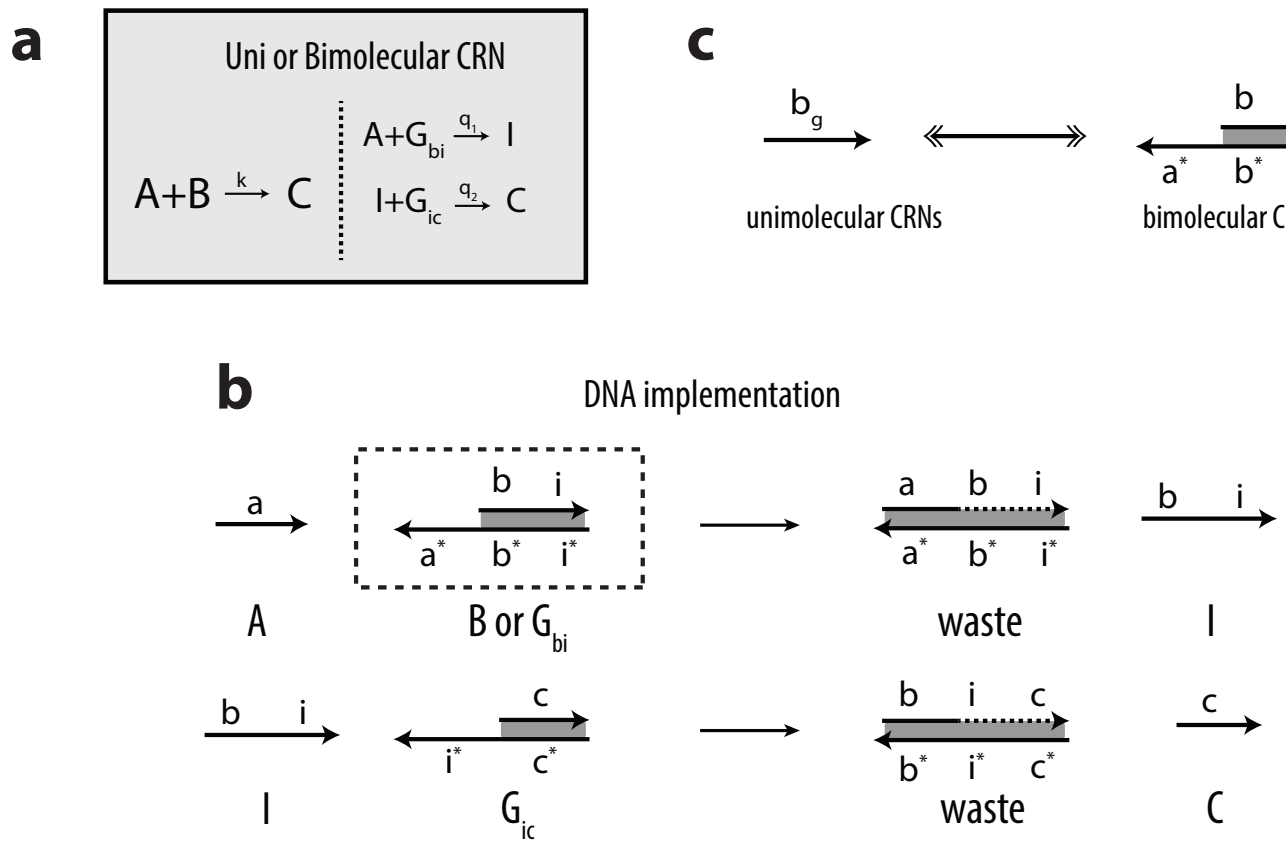

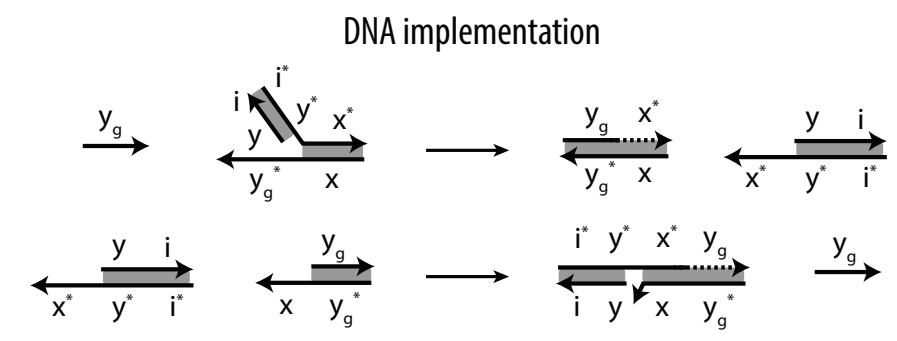

Figure S2: DNA implementation of a unimolecular and bimolecular CRN. a A simple bimolecular CRN consumes two inputs A and B to produce output C. The corresponding two-step DNA-based CRN implementation. b Domain level design of DNA implementation. The input A combines with gate B to produce intermediate strand I. The intermediate combines with an auxiliary gate to produce output $\mathrm{C}$. The highlighted complex can act as an auxiliary complex if unimolecular $\mathrm{CRN}$ is implemented. c A linker reaction can convert between single stranded input B and gate B if a complex system requires both the forms of input at the same time. (d) Full implementation of the linker CRN which can intercovert a specie $\mathrm{Y}$ between its gated form and single stranded form.

gates with same input and output domains cannot be annealed.

Assuming that the auxiliary gates are in excess w.r.t inputs, it can be proved that our DNA implementation can approximate the desired unimolecular and bimolecular CRNs. ${ }^{1-3}$ The final missing block of the system would be a linker reaction ${ }^{2}$ which can interconvert input B between single-stranded and gated form if a complex set of CRNs requires input B be part of both unimolecular and bimolecular CRN (refer to supplementary Fig. S2c and 
Fig. S2d). Full details of the linker reaction implementation and the theory to approximate reaction rates can be found in the theory work Shah et al. (2019). ${ }^{2}$

\section{Reporter calibration}
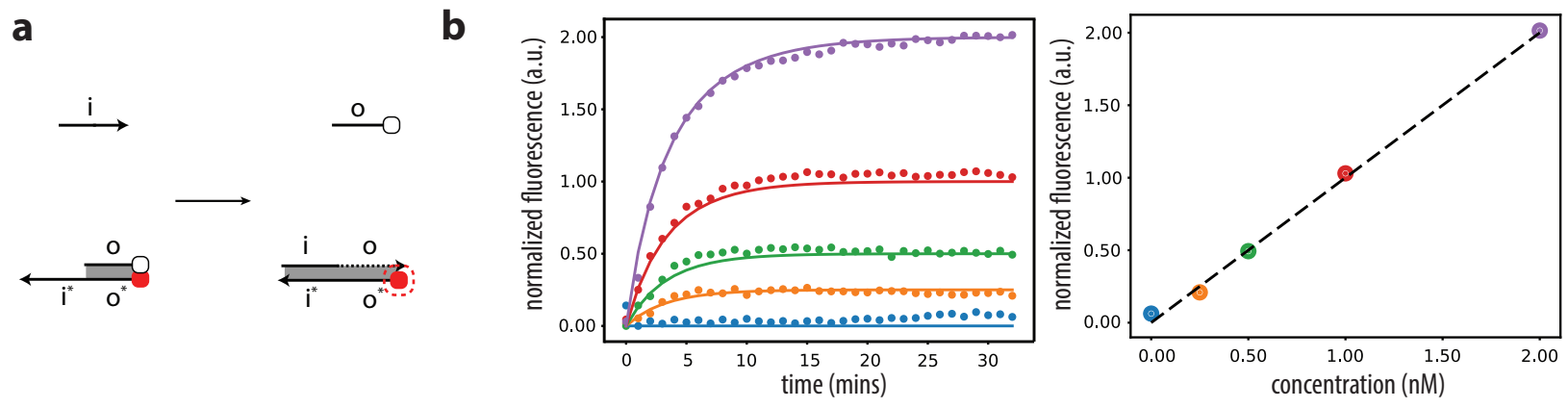

input concentration at $50^{\circ} \mathrm{C}$

$1.00 x$

$0.50 x$

$0.25 x$

Figure S3: Fluorescence calibration curve at $50^{\circ} \mathrm{C}$. a Normalized fluorescence date for different concentrations of input strand $(0.0 \mathrm{x}-2.0 \mathrm{x})$ keeping reporter concentration excess at $5.0 \mathrm{x}$, where $1.0 \mathrm{x}=1 \mathrm{nM}$. b An orthogonal plot showing the saturation normalized fluorescence vs input concentration which demonstrates roughly a linear relationship between them.

The standard reporting method in DNA nanoscience includes the use of fluorescence spectroscopy and a dsDNA complex with fluorescence quencher pair. We also use a doublestranded reporter complex with an exposed toehold region. Upon strand displacement, the quencher strand gets displaced and leads to fluorescence. It should be noted that displace-

ment of quencher strand instead of fluorescence strand is an important design decision as it allows us to avoid non-denaturing purification of the annealed reporter complex. To test the reporter, a fluorescence calibration control experiment was performed with different concentrations of input, as shown in Fig. S3. The normalized fluorescence curves show a linear separation of the steady state fluorescence data and therefore, we use reporter concentrations to normalize the raw fluorescence curves. 

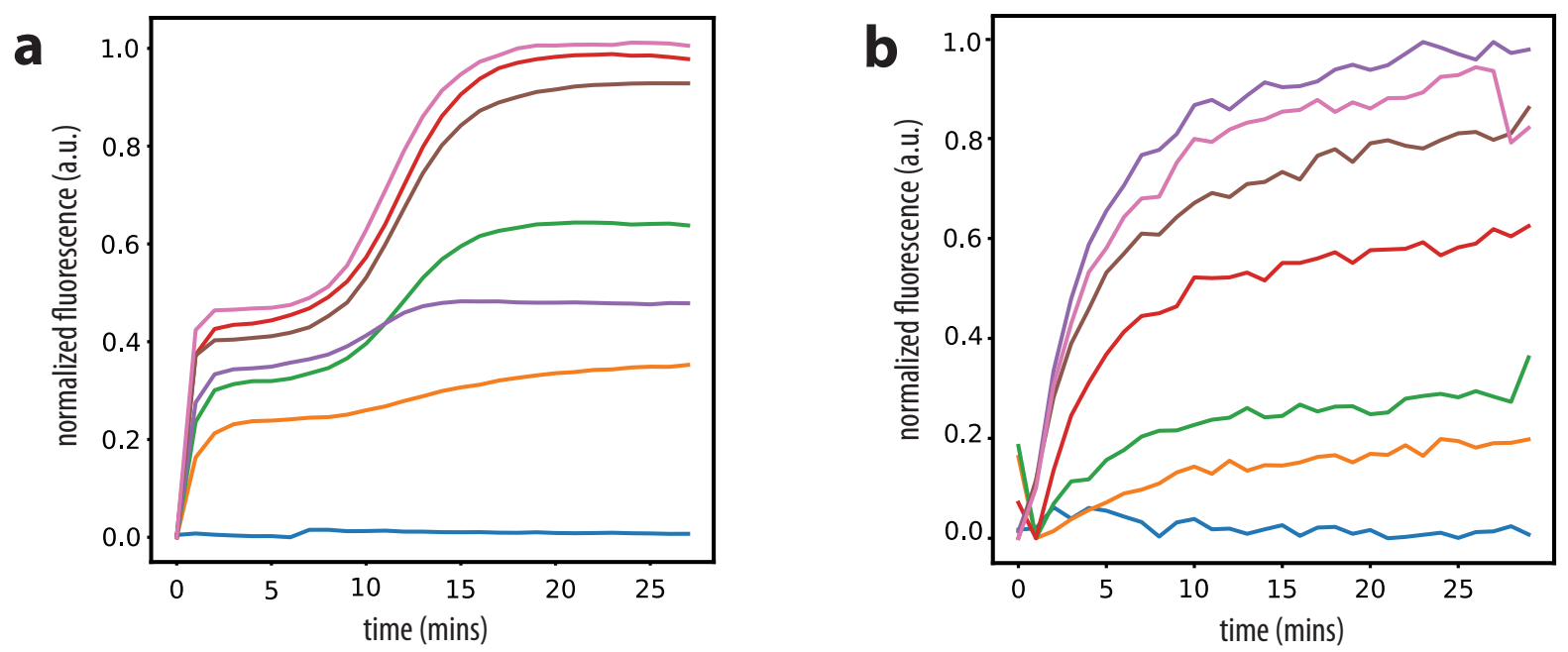

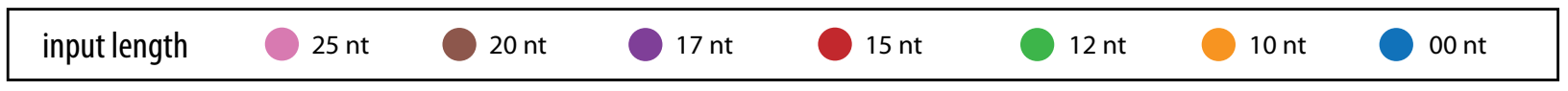

Figure S4: Incubation period for operating at higher temperatures. a A delayed polymerase activity is observed when there is excess temperature fluctuation. For example, adding a relatively high volume of input (15\% of sample volume, input concentration $1.0 \mathrm{x}=10$ $\mathrm{nM})$ at RT to incubated master mix $\left(50^{\circ} \mathrm{C}\right)$. b Expected activity of polymerase activation and strand displacement when the input volume is minimal (1\% of sample volume, input concentration $1.0 \mathrm{x}=1 \mathrm{nM})$.

\section{Sensitivity of polymerase enzyme w.r.t temperature}

In this work, we use the strand displacing polymerase enzyme Bst 3.0. The temperature dependence of the polymerase enzyme is well-known and the recommended temperature of operation is around $65^{\circ} \mathrm{C}$. However, to prevent overly long domain lengths, high DNA synthesis cost and poor yield, we targeted our reaction temperature to be $50^{\circ} \mathrm{C}$. This is much lower than the ideal temperature of polymerase enzyme, however, its activity is still strong (temperature data not shown).

The other thing we noticed was a delayed activity of polymerase enzyme due to temperature difference. Our early reaction mix included adding $15 \mu \mathrm{l}$ of input (15\% of total volume, $1.0 \mathrm{x}=10 \mathrm{nM}$ ) at room temperature to $85 \mu \mathrm{l}$ of master mix that contains polymerase incubated at $50^{\circ} \mathrm{C}$. Although the process of adding input was quick, adding input at room temperature lead to temperature variance of the overall sample and therefore delayed activity of polymerase enzyme as seen in Fig. S4a. Such varying polymerase activity was 
greatly mitigated when the amount of input added at room temperature was reduced to $1 \mu l$ ( $1 \%$ of total volume, $1.0 \mathrm{x}=1 \mathrm{nM})$. Total sample volume for both the experiments was kept constant at $100 \mu l$.

\section{Clamping strategy to reduce reaction leak}

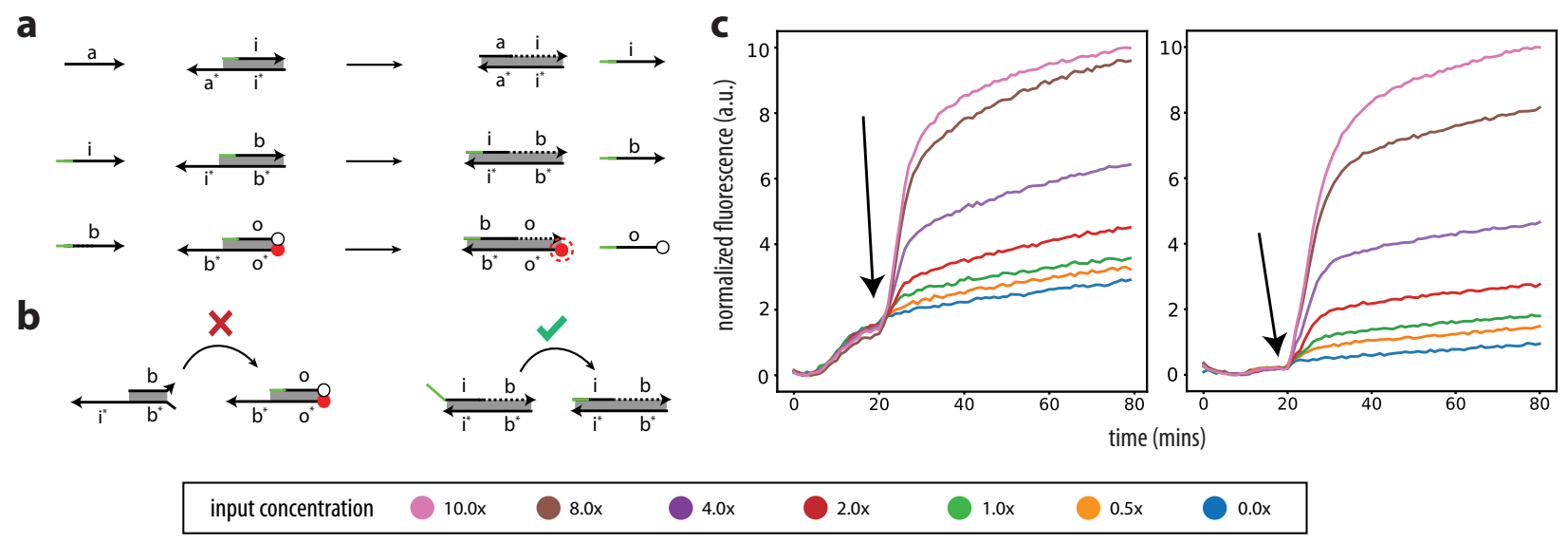

Figure S5: Clamping complexes to reduce leak. a A short 5 nt clamp was added (shown in green) at the $5^{\prime}$ end of each complex to avoid breathing related leaks. b Leaks at $3^{\prime}$ end can open down stream complex and therefore we want to avoid them. Dangling strand at $5^{\prime}$ end does not affect reaction leak since polymerase will blunt it out. c Fluorescence curves demonstrating clamping reduced activation before inputs are added (20 minutes) to the sample.

The control experiments to test a two-step implementation of a CRN produced a signal leak. An example fluorescence curve without $\mathrm{x}$-axis normalization demonstrating the initial leak effect is shown in Fig. S5c. For most of the fluorescence curves shown in this work, the numerical zero for time axis is when input is added to the solution. However, as we observe from the left sub-graph of Fig. S5c, a certain amount of output gets released even before input is added (initial leak) and there is a slow rate of leak during the reaction progression (gradual leak). ${ }^{4}$ To mitigate this effect, we introduced the strategy of $5^{\prime}$ clamping on down stream gates, shown as green domains in Fig. S5a. Such downstream clamping avoids the reaction leak due to breathing effect, for example between complex ib and reporter bo in Fig. S5b. Additionally, no side effects are observed by such additional domains on $5^{\prime}$ end 
since polymerase usually blunts them. The output fluorescence data after $5^{\prime}$ clamping is shown in the right sub-graph of Fig. S5c.

\section{$7 \quad$ Inhibiting the polymerase activity}
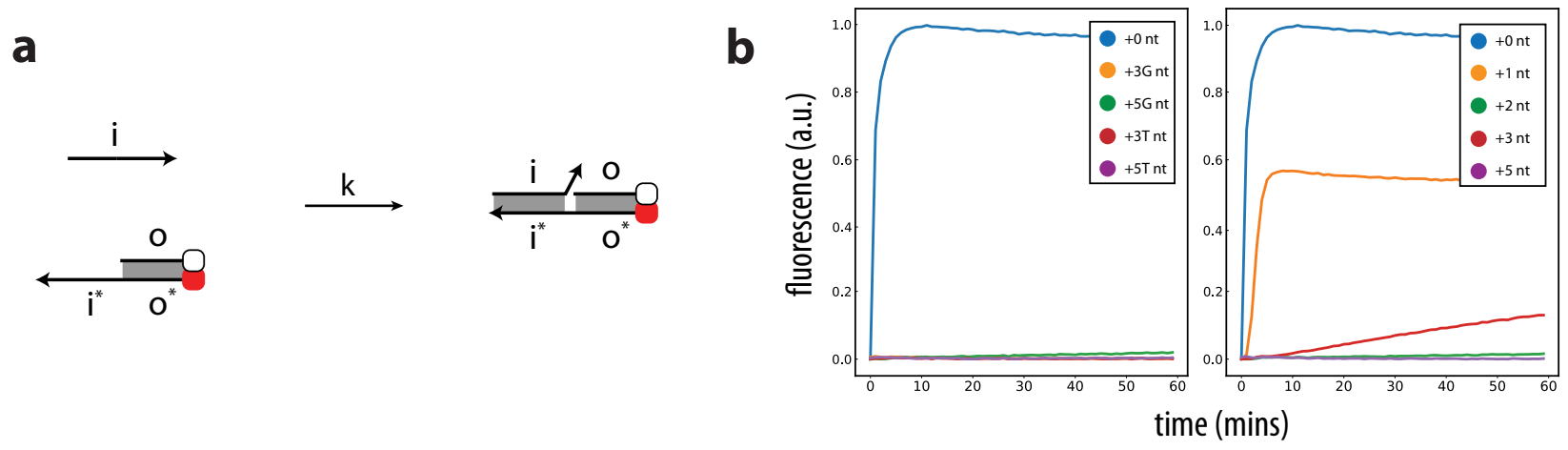

Figure S6: Control experiment to test polymerase activity with $3^{\prime}$ overhang. a An input strand contains complementary sequence with mismatches at the $3^{\prime}$ end. Such overhang should avoid polymerase from priming the strand and displacing quencher strand. b Fluorescence data with different combinations of $3^{\prime}$ mismatches: poly- $\mathrm{T}$, poly-G, and random-N sequences were tried.

An important aspect to implement arbitrary CRNs is multi-output reactions. However, to design multi-output complex, two incumbent strands are required. For such complex to avoid PSD, a $3^{\prime}$ overhang is required as it is well-known that primer-template mismatches greatly reduce the efficiency of polymerase amplification. ${ }^{5}$ Therefore, we tried a few different sets of overhang sequences such as poly- $\mathrm{T}$, poly-G and random N-mer. While the general trend showed that as we increase the size of overhang (or mismatch), the polymerase activity decreases, there was an anomaly at 3 nt (refer to supplementary Fig. S6b). Although this phenomenon can be explained by carefully looking at sequence around mismatches, it means that the random N-mer method requires a careful design of the DNA sequences. In contrast, the poly- $\mathrm{T}$ and poly-G overhangs can effectively stop polymerase activity without anomalies, as seen in supplementary Fig. S6b. Therefore, we simply use a poly-T tail for all our designs that requires inhibiting the polymerase enzyme from its priming activity. 


\section{DNA domains and sequences}

Table S1: Domain names and sequences for tuning reaction speed and rate sequences

\begin{tabular}{||l|l||}
\hline Domain name & Sequence $\left(\mathbf{5}^{\prime}\right.$ to $\mathbf{3}^{\prime}$ direction $)$ \\
\hline $\mathrm{X} 1$ & CACACTTCAAACTCACAACTCAAACATACA \\
\hline $\mathrm{X} 1 *$ & TGTATGTTTGAGTTGTGAGTTTGAAGTGTG \\
\hline $\mathrm{R} 1$ & CACTTCACAACTACACAACAAACCATTACACG \\
\hline $\mathrm{R} 1^{*}$ & CGTGTAATGGTTTGTTGTGTAGTTGTGAAGTG \\
\hline
\end{tabular}

Table S2: Domain-level representation for the DNA sequence

\begin{tabular}{||l|l||}
\hline Name & Domains $\left(5^{\prime}\right.$ to $\mathbf{3}^{\prime}$ direction $)$ \\
\hline Input $(10 \mathrm{nt})$ & $\mathrm{X} 1[21: 30]$ \\
\hline Input $(12 \mathrm{nt})$ & $\mathrm{X} 1[18: 30]$ \\
\hline Input $(15 \mathrm{nt})$ & $\mathrm{X} 1[16: 30]$ \\
\hline Input $(17 \mathrm{nt})$ & $\mathrm{X} 1[13: 30]$ \\
\hline Input (20 nt) & $\mathrm{X} 1[11: 30]$ \\
\hline Input (25 nt) & $\mathrm{X} 1[6: 30]$ \\
\hline Input (30 nt) & $\mathrm{X} 1$ \\
\hline FluorS & $/ 56-F A M / R 1^{*} \mathrm{X} 1^{*}$ \\
\hline QnchS & $\mathrm{R} 1 / 3 \mathrm{BHQ} 1 /$ \\
\hline
\end{tabular}


Table S3: Domain names and sequences for the three-step unimolecular CRN

\begin{tabular}{||l|l||}
\hline Domain name & Sequence $\left(\mathbf{5}^{\prime}\right.$ to $\mathbf{3}^{\prime}$ direction $)$ \\
\hline $\mathrm{X} 1$ & CACACTTCAAACACTCAAACATACA \\
\hline $\mathrm{X} 1{ }^{*}$ & TGTATGTTTGAGTGTTTGAAGTGTG \\
\hline $\mathrm{X} 2{ }^{*}$ & CACATAACCACACTCTCCATCA TTT \\
\hline $\mathrm{X} 3$ & AAA TGATGGAGAGTGTGGTTATGTG \\
\hline $\mathrm{X} 3 *$ & CATCCACTCACACCACCAAACTTCA \\
\hline $\mathrm{X} 4$ & TGAAGTTTGGTGGTGTGAGTGGATG \\
\hline $\mathrm{X} 5^{*}$ & CATCAATCAACACAACTCATTACCA \\
\hline $\mathrm{R} 1$ & TGGTAATGAGTTGTGTTGATTGATG \\
\hline $\mathrm{R} 1 *$ & CACTTCACAACAACACCATTACACG \\
\hline $\mathrm{R} 2$ & CGTGTAATGGTGTTGTTGTGAAGTG \\
\hline $\mathrm{R} 2 *$ & CACTAACATACAACCAACATCTACA \\
\hline
\end{tabular}

Table S4: Domain-level representation for the three-step unimolecular CRN

\begin{tabular}{||l|l||}
\hline Name & Domains $\left(5^{\prime}\right.$ to $\mathbf{3}^{\prime}$ direction $)$ \\
\hline input A & $\mathrm{X} 4$ \\
\hline intd I & $\mathrm{X} 4[21: 25] \mathrm{X} 3$ \\
\hline output B & $\mathrm{X} 2$ \\
\hline output C & $\mathrm{X} 1$ \\
\hline gate AI & $\mathrm{X} 3^{*} \mathrm{X} 4^{*}$ \\
\hline gate IBC & $\mathrm{X} 1^{*} \mathrm{X} 2 *[4: 25] \mathrm{X} 3^{*}$ \\
\hline gate IBA & $\mathrm{X} 4^{*} \mathrm{X} 2 *[4: 25] \mathrm{X} 3^{*}$ \\
\hline FluorS B & $/ 56-\mathrm{FAM} / \mathrm{R} 2^{*} \mathrm{X} 2^{*}$ \\
\hline QnchS B & $\mathrm{X} 2[21: 25] \mathrm{R} 2 / 3 \mathrm{BHQ} 1 /$ \\
\hline FluorS B & $/ 56-\mathrm{FAM} / \mathrm{R} 1^{*} \mathrm{X} 1^{*}$ \\
\hline QnchS B & $\mathrm{X} 1[21: 25] \mathrm{R} 1 / 3 \mathrm{BHQ} 1 /$ \\
\hline
\end{tabular}




\section{References}

(1) Soloveichik, D.; Seelig, G.; Winfree, E. DNA as a universal substrate for chemical kinetics. Proceedings of the National Academy of Sciences 2010, 107, 5393-5398.

(2) Shah, S.; Song, T.; Song, X.; Yang, M.; Reif, J. Implementing arbitrary CRNs using strand displacing polymerase. International Conference on DNA Computing and Molecular Programming. 2019; pp 21-36.

(3) Chen, Y.-J.; Dalchau, N.; Srinivas, N.; Phillips, A.; Cardelli, L.; Soloveichik, D.; Seelig, G. Programmable chemical controllers made from DNA. Nature nanotechnology 2013, 8, 755 .

(4) Srinivas, N.; Parkin, J.; Seelig, G.; Winfree, E.; Soloveichik, D. Enzyme-free nucleic acid dynamical systems. Science 2017, 358, eaal2052.

(5) Kwok, S.; Kellogg, D.; McKinney, N.; Spasic, D.; Goda, L.; Levenson, C.; Sninsky, J. Effects of primer-template mismatches on the polymerase chain reaction: human immunodeficiency virus type 1 model studies. Nucleic acids research 1990, 18, 999-1005. 